\title{
Bonding of Y-TZP to Dentin: Effects of Y-TZP Surface Conditioning, Resin Cement Type, and Aging
}

\author{
MA Bottino • C Bergoli • EG Lima \\ SMS Marocho $\bullet$ RO Souza $\bullet$ LF Valandro
}

\begin{abstract}
Clinical Relevance
The application of the low-fusing glaze porcelain followed by hydrofluoric acid etching and silanization and tribochemical silicatization generates strong bonds between resin cement and zirconia. Panavia generates stronger bonds than does Clearfil.
\end{abstract}

Marco Antonio Bottino, DDS, PhD, chair and professor, MScD-PhD Graduate Program in Restorative Dentistry, Prosthodontic Unit, Science and Technology Institute, Dental School, São Paulo State University (UNESP), São José dos Campos/SP, Brazil

César Dalmolin Bergoli, DDS, MScD, PhD student in Prosthodontics, Graduate Program in Restorative Dentistry, Science and Technology Institute, Dental School, São Paulo State University (UNESP), São José dos Campos/SP, Brazil.

Elen Guerra Lima, DDS, MScD student in Prosthodontics, Graduate Program in Restorative Dentistry, Science and Technology Institute, Dental School, São Paulo State University (UNESP), São José dos Campos/SP, Brazil.

Susana María Salazar Marocho, DDS, MScD, PhD, Science and Technology Institute, Dental School,São Paulo State University (UNESP), São José dos Campos/SP, Brazil.

Rodrigo Othavio Assunção Souza, DDS, MScD, PhD, adjunct professor, Federal University of Paraíba (UFPB), Department of Restorative Dentistry, Division of Prosthodontics, João Pessoa/Paraíba, Brazil.

*Luiz Felipe Valandro, DDS, MScD, PhD, associate professor, Federal University of Santa Maria (UFSM), Head of MSciDPhD Graduate Program in Oral Science, Prosthodontic Unit, Faculty of Odontology, Santa Maria, RS, Brazil.

*Corresponding author: R. Floriano Peixoto 1184, Santa Maria, RS 97015-372, Brazil; e-mail: lfvalandro@hotmail. com

DOI: $10.2341 / 12-235-\mathrm{L}$

\section{SUMMARY}

Purpose: To evaluate the effects of two surface treatments, aging, and two resin cements on shear bond strength between dentin and yttrium-stabilized tetragonal zirconia polycrystal ceramic (Y-TZP).

Materials and Methods: Eighty human molars were embedded in acrylic resin and sectioned $3 \mathrm{~mm}$ below the occlusal plane. These teeth and 80 cylindrical Y-TZP specimens (height, $4 \mathrm{~mm}$; diameter, $3.4 \mathrm{~mm}$ ) were divided into eight groups $(n=10)$ using the following factors: $Y$ TZP surface treatment (Vi: low-fusing porcelain [vitrification] + hydrofluoric acid etching + silanization or Si: tribochemical silicatization); cementation strategies (PF: Panavia or CC: Clearfil); and storage (nonaging or aging). Bonding surfaces of 40 Y-TZP specimens received Vi treatment, and the rest received $\mathrm{Si}$ treatment. Half of the ceramic-tooth assemblies were cemented with Panavia, the rest with Clearfil. Shear tests were executed using 0.4-mm-thick wire at $0.5 \mathrm{~mm} / \mathrm{min}$. Data were analyzed by three-way analysis of variance and Tukey test $(\alpha=0.05)$. Fractures were analyzed. 
Results: Y-TZP surface treatments did not affect bond strength $(p=0.762, \mathrm{Vi}=\mathrm{Si})$, while resin cements $(p<0.001$, Panavia $>$ Clearfil $)$ and aging ( $p=0.006$, nonaging $>$ aging) showed a significant effect. Most failures were in adhesive at dentin-cement interfaces; no failure occurred between zirconia and cement.

Conclusion: When Y-TZP ceramic is bonded to dentin, the weakest interface is that between dentin and resin cement. The resin cement/Y-TZP interface was less susceptible to failures, owing to Y-TZP surface treatments.

\section{INTRODUCTION}

Zirconia-based dental ceramics have better mechanical properties than do other commercially available ceramic materials. ${ }^{1}$ However, their bond strength to resin cement has been reported ${ }^{2}$ to be weak because zirconia-based ceramics have a large crystalline phase, rendering conventional hydrofluoric acid etching treatment impossible. Therefore, in the last few years, many researchers ${ }^{2-6}$ have studied alternative methods of improving the adhesion between zirconia and resin cements.

Among the possible treatments being investigated to improve the adhesion, air-particle abrasion protocols have attracted much interest because of their simplicity. These methods are called tribochemical silicatization and involve air abrasion of the ceramic surface with alumina or with silica-modified alumina particles $(30 \mu \mathrm{m})$, followed by the application of a silane coupling agent. ${ }^{1,7-9}$ The alumina- or silicamodified alumina particles produce microroughness on the zirconia surface, while the silane coupling agent acts as a link between the sandblasted surface and the cement matrix..$^{8-13}$

In addition to the air-abrasion protocols, resin cements containing phosphate ester monomer 10methacryloyloxydecyl dihydrogen phosphate (MDP) have been shown to form strong bonds with zirconia. These cements react with oxides, creating a good interaction with the zirconia surface. ${ }^{8,10-13}$ However, a study ${ }^{10}$ has showed that the use of cements with MDP alone is not sufficient for creating a stable union between the resin cement and zirconia. Therefore, air-abrasion protocols ${ }^{14}$ are also required to be a part of the bonding technique. On the other hand, airborne particle abrasion procedures can structurally damage the zirconia surface, decreasing the mechanical properties of this ceramic ${ }^{15}$; hence, the use of these procedures remains controversial.
As a new alternative to the surface treatment of zirconia, some researchers ${ }^{4,16-18}$ have evaluated the application of a low-fusing porcelain material (glaze) on the yttrium-doped tetragonal zirconia (Y-TZP) intaglio surface. This technique aims to create a surface that can be etched by hydrofluoric acid, similar to feldspathic ceramics. Although this is a promising technique, it has been introduced very recently, and data on resin bond strength and bond durability as a result of using this method are scarce.

In fixed dental prostheses (FDPs), in which mechanical retention plays an important role, the mechanism responsible for adhesion with dentin appears to be less important. ${ }^{19}$ On the other hand, in bonded FDPs for which mechanical retention is not the primary retention mechanism the bond strength between the resin cement and dentin is essential to the success of the treatment. ${ }^{20,21}$ Hence, evaluation of the adhesion between Y-TZP, resin cements, and dentin is important. ${ }^{5,22}$

Chai and others ${ }^{5}$ reported that studies that evaluate simply the bond strength between resin cements and restorative materials are limited in scope from a clinical perspective, as FDP restorations are normally cemented to an enamel/dentin substrate. When zirconia specimens are cemented onto a dental substrate, it is possible to assess both interfaces, such as the cement-ceramic and cementdentin interfaces, which leads to better evaluation of adhesion, from the clinical point of view.

Therefore, the aim of the present study is to evaluate two cementation strategies (involving the use of the resin cements Panavia F and Clearfil SA Cement, respectively), two Y-TZP surface treatment techniques (involving the application of a low-fusing porcelain and silicatization, respectively), and the effect of thermocycling aging on the shear bond strength between zirconia and dentin. The following hypotheses were tested: 1) the application of a low-fusing glaze porcelain application and silanization as Y-TZP surface treatments will not influence the bond strength;2) the cementation strategies will not influence the bond strength; and 3) aging will decrease the bond strength.

\section{MATERIALS AND METHODS}

The product names, manufacturers, chemical compositions, and batch numbers of the materials used in the study are listed in Table 1.

\section{Selection of Teeth}

Eighty human molars were selected, cleaned with periodontal curettes, and stored in distilled water 


\begin{tabular}{|c|c|c|c|}
\hline Material & Manufacturer & Chemical Composition & Batch No. \\
\hline Rocatec Plus & 3M ESPE, Seefeld, Germany & Silicatized aluminum oxide particles $(30 \mu \mathrm{m})$ & 1036301855 \\
\hline Porcelain Conditioner & Dentstply, Petrópolis, RJ, Brazil & $10 \%$ Hydrofluoric acid & 229431B \\
\hline Vita Akzent Glaze Spray & Vita Zahnfabrick, Germany & Not available & 21790 \\
\hline Clearfill SA Cement & Kuraray Medical Inc, Japan & $\begin{array}{l}\text { Bis-GMA, TEGDMA, MDP, hydrophobic aromatic } \\
\text { dimethacrylate, silanated barium glass filler, silanated } \\
\text { colloidal silica, di-camphorquinone, benzoyl peroxide, } \\
\text { initiator, hydrophobic aliphatic dimethacrylate, } \\
\text { silanated, surface-treated sodium fluoride, } \\
\text { accelerators, pigments, } 45 \mathrm{vol} \% \text { is inorganic fillers }\end{array}$ & 023AAB \\
\hline Monobond S & $\begin{array}{l}\text { Ivoclar Vivadent, Schaan, } \\
\text { Liechtenstein }\end{array}$ & Alcohol solution of silane metacrylate & 532888 \\
\hline Panavia F-Cement Paste A & Kuraray Medical Inc, Japan & $\begin{array}{l}\text { 10-Methacryloyloxydecyl dihydrogen phosphate, } \\
\text { hydrophobic aromatic dimethacrylate, hydrophobic } \\
\text { aliphatic methacrylate, hydrophilic aliphatic } \\
\text { dimethacrylate, silanated silica filler, silanated } \\
\text { colloidal silica, DL-camphorquinone, catalysts, } \\
\text { initiators, others }\end{array}$ & 00251B \\
\hline Panavia F-Cement Paste B & Kuraray Medical Inc, Japan & $\begin{array}{l}\text { Sodium fluoride, hydrophobic aromatic } \\
\text { dimethacrylate, hydrophobic aliphatic methacrylate, } \\
\text { hydrophilic aliphatic dimethacrylate, silanated barium } \\
\text { glass filler, catalysts, accelerators, pigments, others }\end{array}$ & 00029A \\
\hline Vita In Ceram YZ & Vita Zahnfabrick, Germany & $\begin{array}{l}\text { 91\% Zirconium oxide }\left(\mathrm{ZrO}_{2}\right), 5 \% \text { yttrium oxide }\left(\mathrm{Y}_{2} \mathrm{O}_{3}\right) \text {, } \\
3 \% \text { hafnium oxide }\left(\mathrm{HfO}_{2}\right) \text {, small amounts }(<1 \%) \text { of } \\
\text { aluminum oxide }\left(\mathrm{Al}_{2} \mathrm{O}_{3}\right) \text { and silicon oxide }\left(\mathrm{SiO}_{2}\right)\end{array}$ & 28070 \\
\hline
\end{tabular}

$\left(4^{\circ} \mathrm{C}\right)$ until needed. With the assistance of a cylindrical metallic mold (diameter, $20 \mathrm{~mm}$; height, $15 \mathrm{~mm}$ ), each tooth was embedded $2 \mathrm{~mm}$ apical to the cemento-enamel junction with a self-curing acrylic resin (JET, Artigos Odontológicos Classico Ltda, Sao Paulo, Brazil).

Then, each tooth was sectioned $3 \mathrm{~mm}$ below the occlusal surface using a low-speed diamond cutting saw (Labcut 1010, Extec, Enfield, CT, USA) with extensive water cooling in order to expose the superficial coronal dentin surface. These surfaces were then wet-ground with 600-grit silicon paper for 60 seconds using a polishing machine (PSK-2V, Skill TEC, Sao Paulo, Brazil).

Before the cementation procedures, the teeth were numbered from 1 to 80 , and eight random sequences consisting of 10 numbers each were generated using the computer program Random Allocator (developed by M Saghaei, Dept of Anesthesia, Isfahan University of Medical Sciences, Isfahan, Iran). This procedure was performed to homogenize the groups and randomize the allocation of the specimens to the eight groups. ${ }^{23}$ Next, each of the eight groups, which comprised 10 samples each, was assigned to one of the following categories: Y-TZP surface treatment (Vi: application of the low-fusing glaze porcelain [vitrification] + hydrofluoric acid etching + silanization or Si: tribochemical silicatization), cementation strategies (PF: Panavia or CC: Clearfil), and storage condition (nonaging or aging): $\mathrm{Si}+\mathrm{PF} ; \mathrm{Si}+\mathrm{PF}+$ aging; $\mathrm{Si}+\mathrm{CC} ; \mathrm{Si}+\mathrm{CC}+$ aging; $\mathrm{Vi}+\mathrm{PF} ; \mathrm{Vi}+\mathrm{PF}+$ aging; $\mathrm{Vi}+\mathrm{CC}$; and $\mathrm{Vi}+\mathrm{CC}+$ aging.

\section{Preparation of the Y-TZP Specimens}

First, blocks of Vita In Ceram YZ 2000 (Vita Zahnfabrik, Bad Säckingen, Germany) were sectioned using a diamond saw (Labcut 1010, Extec) to produce smaller cubes $\left(5 \times 15 \times 20 \mathrm{~mm}^{3}\right)$. A drill-type trephine was used to perforate these cubes perpendicular to the surface (with the aid of a preparation device) to produce presintered zirconia cylinders (diameter, $4.5 \mathrm{~mm}$; height, $5 \mathrm{~mm}$ ) that were then 
sintered, as recommended by the manufacturer, in an oven (Vita Zyrcomat, Vita Zahnfabrik). The final dimensions of the ceramic cylinders were $3.4 \mathrm{~mm}$ in diameter and $4 \mathrm{~mm}$ in height.

The surface of each cylinder that had to undergo cementation was polished with 800-, 1000-, and 1200-grit silicon carbide paper, under water cooling, for 60 seconds each using a polishing machine (PSK2V, Skill TEC). After polishing, the cylinders were cleaned ultrasonically for five minutes in isopropyl alcohol.

\section{Conditioning of the Y-TZP Surfaces}

For $50 \%$ of the zirconia cylinders $(\mathrm{N}=40)$, a lowfusing porcelain glaze (Vita Akzent Glaze Spray, Vita Zahnfabrik) was applied for one to two seconds on the cementation surface at a $10-\mathrm{mm}$ distance. The conditioned specimen was then sintered (VACUMAT $40 \mathrm{~T}$, Vita Zahnfabrik) according to the manufacturer's instructions. Then the glaze-coated surfaces were treated with 10\% hydrofluoric acid gel (Porcelain Conditioner, Dentsply, Petropolis, RJ, Brazil) for 60 seconds, washed for 15 seconds, dried, and silanized with a methacryloxypropyltrimethoxysilane (MPS)-based silane coupling (Monobond S, Ivoclar Vivadent, Schaan, Liechtenstein). The silanized samples were kept aside for 60 seconds to let the solvent evaporate.

The remaining zirconia cylinders $(\mathrm{N}=40)$ were treated using the tribochemical silicatization method. First, the surfaces of the cylinders were airabraded using $30 \mu \mathrm{m}$ silica-coated alumina particles (Rocatec Soft, 3M ESPE, Seefeld, Germany) from a distance of $10 \mathrm{~mm}$ and with a pressure of 2.8 bar. Subsequently, the MPS-based silane coupling agent (Monobond S, Ivoclar Vivadent) was applied in the manner described above.

\section{Cementation Procedures}

The cementation surface of the specimens was defined by an adhesive tape (Scotch, 3M, Ribeirão Preto, Brazil) with a 3.4-mm-diameter hole, aiming to standardize the cementation area and prevent the overflow of the resin cement.

For the PF samples, the dentinal surface treatment was performed as follows. Equal amounts of Primers A and B were mixed, and this mixture was applied on the dentinal surface with a microbrush. This was followed by spraying the surface gently with air and letting it stand for one minute to allow the reaction to take place. For CC samples, dentinal surface treatment was not required, as the Clearfil SA Cement is self-adhesive.

The two resin cements were manipulated as recommended by the manufacturer and were applied on the conditioned surfaces of the zirconia cylinders. The cylinders were placed on top of the area bounded by the adhesive tape, and a load of $750 \mathrm{~g}$ was applied on the cylinders for 60 seconds. Any excess cement was removed, and all of the surfaces (vestibular, mesial, distal, and lingual) were photoactivated using an LED $\left(1200 \mathrm{~mW} / \mathrm{cm}^{2}\right)$ (Radii Cal, SDI, Australia) for 20 seconds.

\section{Storage Conditions}

All the specimens were stored for 24 hours in distilled water at $37^{\circ} \mathrm{C}$. Half of the specimens were submitted to the shear bond strength test and the other half were aged before testing using a thermocycling protocol that involved 5000 cycles of alternate immersion in baths at $5^{\circ} \mathrm{C}$ and $55^{\circ} \mathrm{C}$ for 30 seconds each with intervals of two seconds between the immersions.

\section{Shear Bond Strength Test}

The test was conducted using a universal testing machine (EMIC DL 1000, Emic, São José dos Pinhais, PR, Brazil) with a crosshead speed of 0.5 $\mathrm{mm} / \mathrm{min}$ and a steel wire with a thickness of $0.4 \mathrm{~mm}$. The test cylinder was aligned with the load cell, and the wire loop was positioned as close as possible to the ceramic/dentin interface and parallel to the direction of the load cell (50 Kgf). The steel wire was then pulled using the universal machine in order to perform the shear bond strength test.

The bond strength was calculated using the formula $R=F / A$, where $R$ is the bond strength ( $\mathrm{MPa}), F$ is the load required for rupture of the specimen $(\mathrm{N})$, and $A$ is the bonded cross-sectional area of the specimen $\left(\mathrm{mm}^{2}\right)$. The bonded crosssectional area was calculated using the formula for the area of a circle, which is given by $A=\pi \times r^{2}$, where $\pi=3.14$ and $r=1.7 \mathrm{~mm}$ (half of the diameter of the cylinder). Using this formula, the bonded cross-sectional area was found to be $9.07 \mathrm{~mm}^{2}$.

\section{Fracture Analysis}

The surfaces of the fractured specimens were examined using an optical stereomicroscope (Discovery V20; Carl Zeiss, Gottingen, Germany) and a scanning electron microscope (SEM; Inspect S50, FEI, Oregon, USA). For the SEM observations, all of the specimens were gold-sputtered under vacuum. 


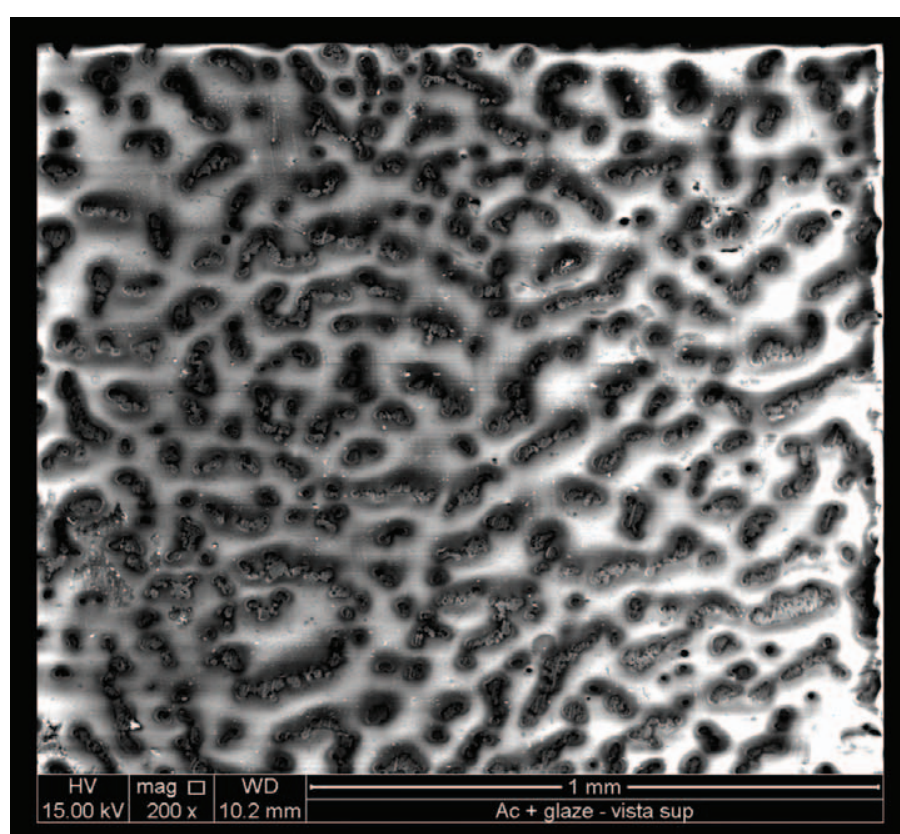

A

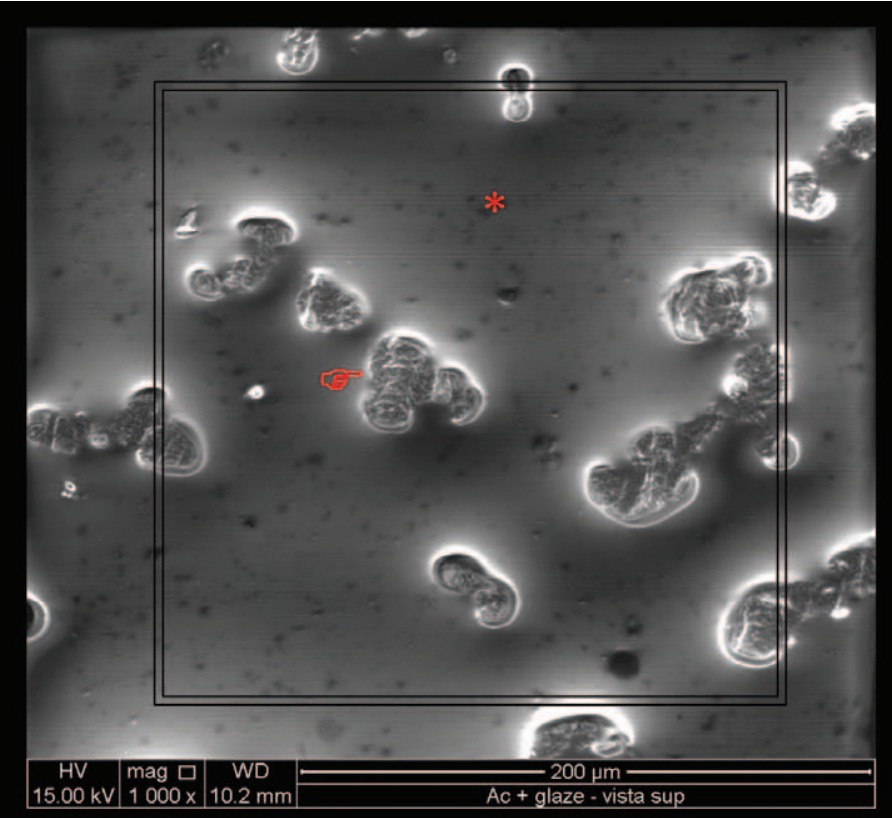

$\mathbf{B}$

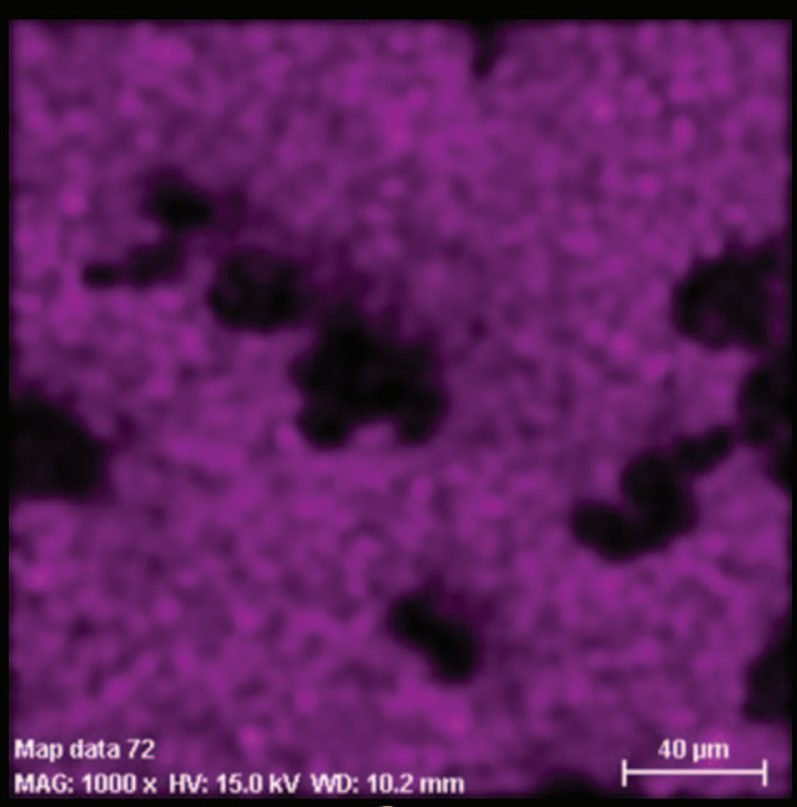

C

Map data 72

MAG: $1000 \times$ HV: $15.0 \mathrm{kV}$ WD: $10.2 \mathrm{~mm}$

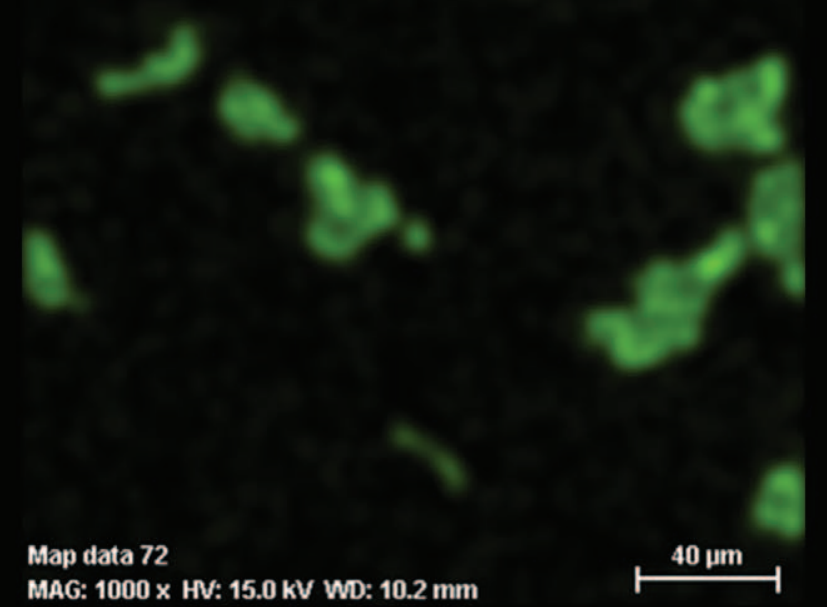

D

Figure 1. (A) Low-fusing porcelain glaze film distribution on zirconia surface at 200x; (B) Glaze distribution at 1000x. The red pointer indicates the area of the zirconia surface that was not covered by glaze film, while the asterisk indicates the silicon surface created by vitrification (the square is the area of Energy Dispesive Scanning [EDS] analysis); (C) Representative EDS element maps acquired (the purple area represents the silicon on the zirconia surface); (D) EDS element maps acquired (the green area represents the zirconium surface that was not covered by silicon).

Fracture analysis was performed for several specimens in order to identify the fracture origin and mode of fracture. Stereomicroscope examinations were performed using various lighting configurations to identify the fracture pattern. The identified fractures were classified on the basis of the following scheme: score $\mathrm{A}=$ a detachment of the resin cement from the dentin; score $\mathrm{B}=\mathrm{a}$ detachment of the ceramic from the resin cement; score $\mathrm{C}=$ a fracture of ceramic without an adhesive failure; score $\mathrm{D}=\mathrm{a}$ 


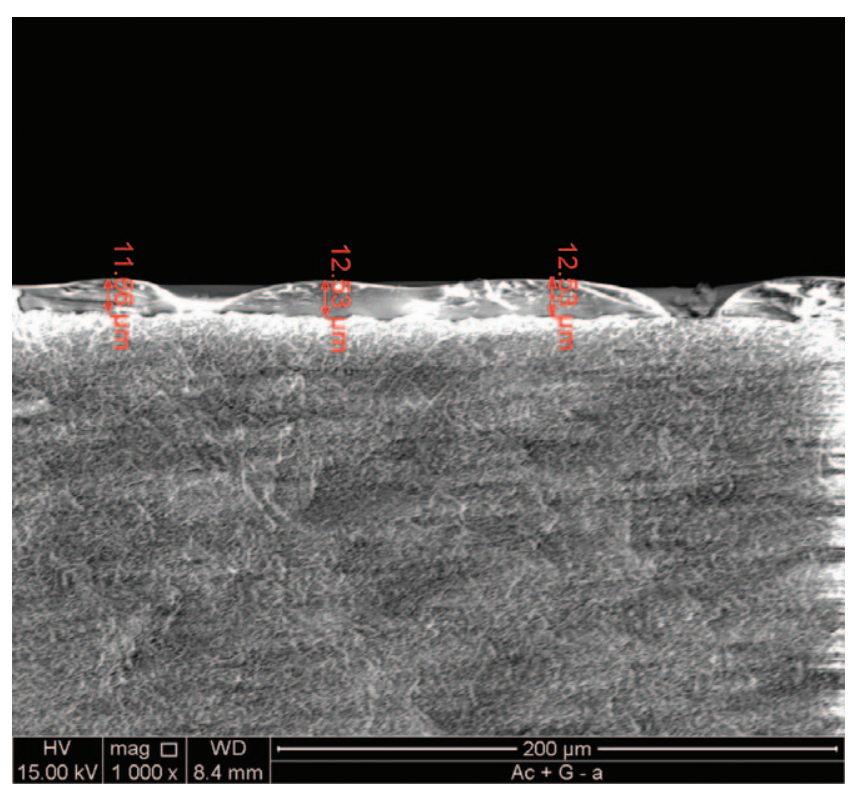

Figure 2. Low-fusing porcelain glaze thickness measured on zirconia surface under SEM.

fracture of dentin, without an adhesive failure; and score $\mathrm{E}=$ an area of resin cement fracture bigger than an area of adhesive failure.

\section{Measurement of Glaze Thickness}

A pilot study was conducted to measure the thickness of the glaze obtained by the vitrification process. To measure the thickness of the glaze layer, three zirconia beams $(10 \times 2 \times 2 \mathrm{~mm})$ were sprayed with the glaze, sintered, and broken into two pieces so that the glaze thickness could be measured by SEM at a magnification of $1000 \times$. As can be noted in Figure 1, the applied glaze is homogeneously distributed on the zirconia surface. In addition, the thickness of the glaze layer was also found to be of an acceptable level, with a mean value of $12 \pm 0.3 \mu \mathrm{m}$ (Figure 2).

\section{Data Analysis}

The bond strength data were analyzed using threeway analysis of variance (ANOVA) and Tukey test $(\alpha=0.05)$ with the software Minitab 16.1.0. The specimens with pretest failures (during the aging process) were included in the statistical analysis and were conferred a bond strength value of 0 $\mathrm{MPa}$.

\section{RESULTS}

The factors resin cement ( $p<0.001$; Panavia $>$ Clearfil) and storage condition ( $p=0.006$; nonaging $>$ aging) statistically influenced the bond strength values, while the factor surface treatment $(p=0.762 ; \mathrm{Vi}=\mathrm{Si})$ had no effect. According to the result of the Tukey test, the groups $\mathrm{Si}+\mathrm{CC}+$ aging $(4.0 \pm 3.4 \mathrm{MPa})$ and $\mathrm{Vi}+\mathrm{CC}+$ aging $(3.9 \pm 3.8$ $\mathrm{MPa})$ exhibited the lowest bond strength values, while the $\mathrm{Vi}+\mathrm{PF}$ group $(17.2 \pm 10.1 \mathrm{MPa})$ showed the highest values of bond strength but was not statistically different than $\mathrm{Vi}+\mathrm{PF}+\mathrm{TC}(14.7 \pm 6.0$ $\mathrm{MPa}), \mathrm{Si}+\mathrm{PF}(14.6 \pm 8.4 \mathrm{MPa}), \mathrm{Si}+\mathrm{PF}+\mathrm{TC}(11.6$ $\pm 6.2 \mathrm{MPa})$, and $\mathrm{Si}+\mathrm{CC}(10.8 \pm 3.8 \mathrm{MPa})$. The group $\mathrm{Vi}+\mathrm{CC}(7.0 \pm 3.4 \mathrm{MPa})$ showed intermediate values. When subjected to the Si surface treatment, the zirconia cylinders cemented with Clearfil cement showed a significant decrease in bond strength values after aging.

Fracture analysis revealed that fractures occurred predominantly in the adhesive at the resin cement/

\begin{tabular}{|c|c|c|c|c|c|c|c|c|c|}
\hline \multirow[t]{2}{*}{ Surface Treatment } & \multirow[t]{2}{*}{ Cement } & \multirow[t]{2}{*}{ Thermo-cycling } & \multirow{2}{*}{$\begin{array}{c}\text { Group } \\
\text { Abbreviations }\end{array}$} & \multirow[t]{2}{*}{ Mean $^{\mathrm{a}}$ (SD) } & \multicolumn{5}{|c|}{ Scores for Failure Type ${ }^{b}$} \\
\hline & & & & & Score A & Score B & Score C & Score D & Score E \\
\hline \multirow{2}{*}{ Tribosilicatization } & Panavia F & Yes & $\mathrm{Si}+\mathrm{PF}+\mathrm{TC}$ & $11.6(6.2) \mathrm{ABC}$ & 4 & 0 & 0 & 0 & 6 \\
\hline & Clearfill SE & No & $\mathrm{Si}+\mathrm{CC}$ & $10.8(3.8) \mathrm{ABC}$ & 6 & 0 & 0 & 0 & 4 \\
\hline \multirow{4}{*}{ Glassy application } & Panavia $\mathrm{F}$ & Yes & $\mathrm{Vi}+\mathrm{PF}+\mathrm{TC}$ & $14.7(6.0) A B$ & 8 & 0 & 0 & 0 & 2 \\
\hline & \multirow[t]{3}{*}{ Clearfill SE } & No & $\mathrm{Vi}+\mathrm{CC}$ & $7.0(3.4) \mathrm{BC}$ & 8 & 0 & 0 & 0 & 2 \\
\hline & & Yes & $\mathrm{Vi}+\mathrm{CC}+\mathrm{TC}$ & $3.9(3.8) \mathrm{c}$ & 7 & 1 & 0 & 0 & 2 \\
\hline & & & & & $52(65 \%)$ & $2(2.5 \%)$ & 0 & 0 & $26(32.5 \%)$ \\
\hline
\end{tabular}




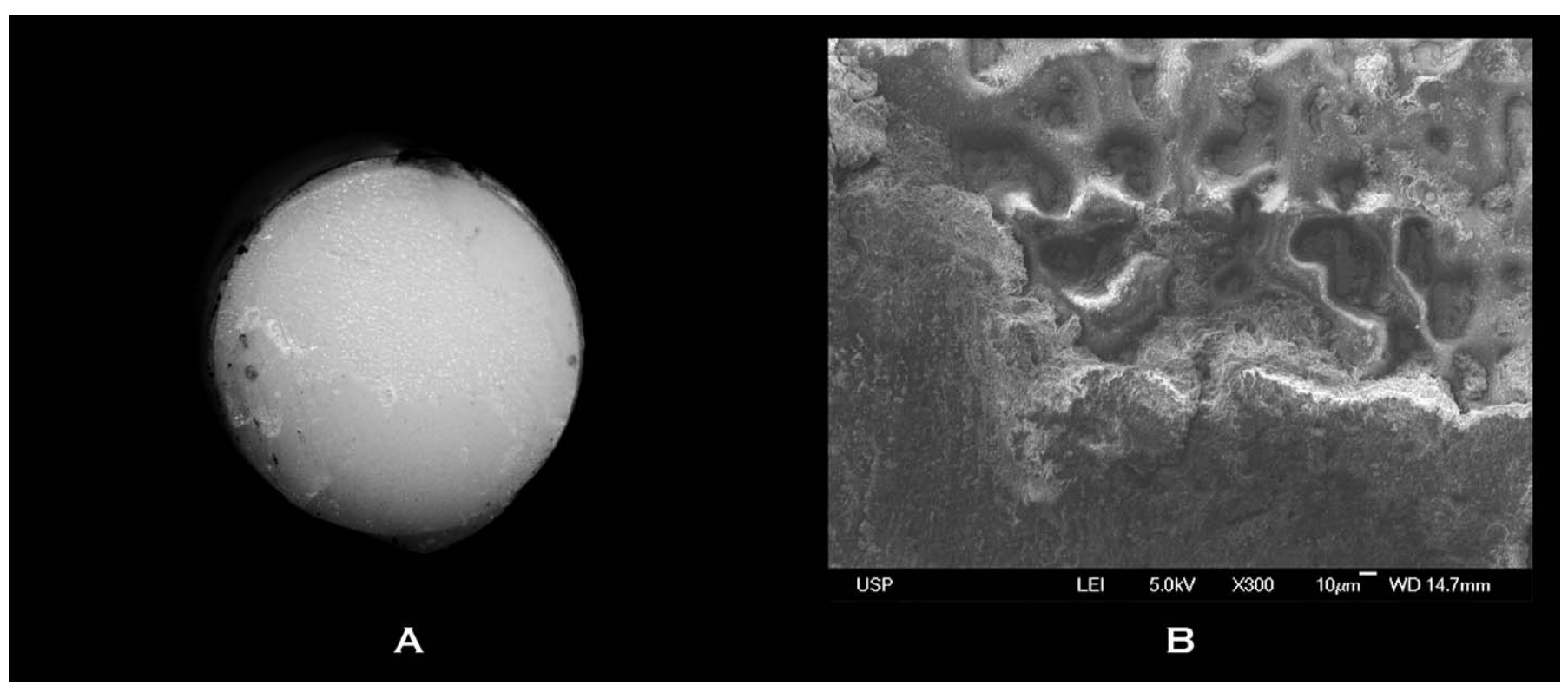

Figure 3. (A) Representative image of a cohesive failure. (B) Detailed view of the small black square in (A). Fracture surface topography revealed the configuration of the glaze film on the zirconia surface, which is associated with exposure of zirconium oxides.

dentin interface $(65 \%$ of the failures were scored type A), followed by cohesive failures in the resin cement (32.5\% were scored type E) and in the adhesive at the resin cement and ceramic interface $(2.5 \%$ were scored type B) (Table 2). Representative images of the different failure modes are shown in Figures 3 and 4. For the groups $\mathrm{Si}+\mathrm{CC}+$ aging and $\mathrm{Vi}+\mathrm{CC}$ + aging, three specimens each failed during aging; for the group $\mathrm{Si}+\mathrm{PF}+$ aging, one specimen failed during aging; and in the group $\mathrm{Vi}+\mathrm{PF}+$ aging, there were no specimen failures during the aging process. All of the premature failures that occurred during aging were at the resin cement and dentin interface (scored type A).

\section{DISCUSSION}

Different surface treatments have been tested to improve the bond strength between resin cement and zirconia. Nowadays, special attention has been given to the treatment of the zirconia intaglio

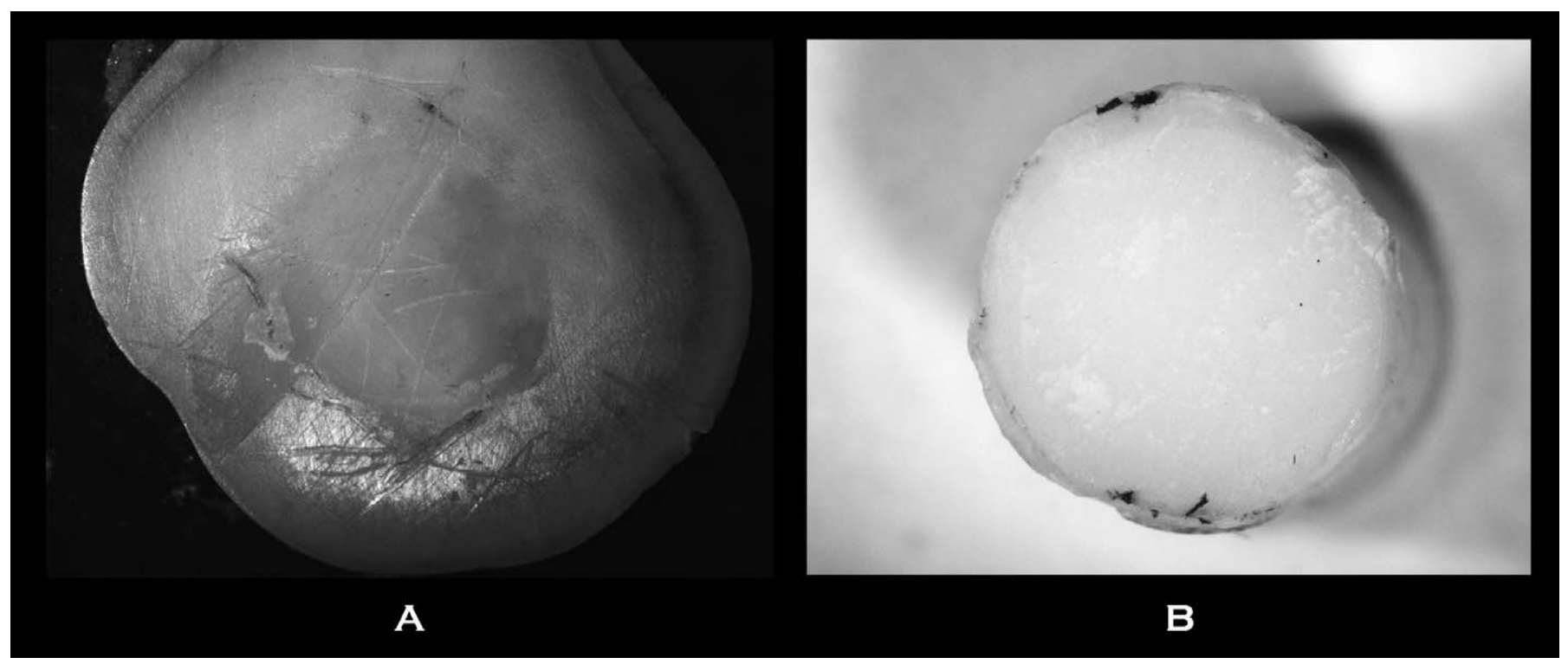

Figure 4. Representative image of a failure at the cement/dentin interface. 
surface with low-fusing porcelain glazes (produced by vitrification), which would create an etchable layer on the acid-resistant material, creating a scenario similar to that found in silica-based ceramics. For studies on zirconia to have clinical relevance, it is important to consider the dentin surface as well. ${ }^{5}$

The three-way ANOVA showed that the zirconia surface treatments resulted in similar bond strengths, confirming the first hypothesis of the study and representing a good pattern of conditioning promoted by Y-TZP surface conditioning approaches. The silicatization after air-abrasion by silica-modified aluminum oxide particles is considered a prerequisite for achieving good adhesion between the resin cement and zirconia surfaces ${ }^{10}$ and leads to the generation of bond strength values that are higher than those obtained from other surface treatments. ${ }^{1,5,7,10}$ This method creates hydroxyl groups and enhances the micromechanical retention of the resins on the zirconia surface. In addition, the application of a silane coupling agent after the silica coating generates a siloxane network that improves the bond between the resin cement and zirconia. ${ }^{10}$ Chai and others ${ }^{5}$ employed an experimental design similar to that used in our study and observed that the tribochemical silica coating of the zirconia surface generated statistically higher values for the bond strength between the zirconia cylinders and the dentin surface. The vitrification method creates an etchable glassy thin film on the acid-resistant zirconia surface. This surface can then be etched by hydrofluoric acid and silanized using an MPS-based primer. In addition, only two specimens subjected to the vitrification process exhibited adhesive failure at the resin cement/zirconia interface. This result corroborates the findings of Cura and others. ${ }^{4}$ On the other hand, in contrast to the results obtained by us, Everson and others, ${ }^{16}$ Valentino and others, ${ }^{17}$ and Ntala and others ${ }^{18}$ observed statistically higher values of bond strength for vitrification techniques than for the tribochemical silica-coating approach. This may be because these studies did not use dentin as an adhesion substrate. We utilized dentin as an adhesion substrate for the zirconia samples, and the majority of the failures occurred at the dentin/resin cement interface, preventing a real evaluation of the adhesion of the resin cement to the treated zirconia surface (comparison between the Y-TZP surface conditioning).

The second hypothesis of the study was rejected, as the bond strength values are statistically affected by the resin cements (Panavia F: 14.5 MPa; Clearfil $\mathrm{SE}$ : $6.4 \mathrm{MPa}$ ). In this study, the resin cement Panavia F showed statistically higher values of shear bond strength than did the self-adhesive resin cement Clearfil SA Cement (Table 2).

According to previously reported studies, ${ }^{24-26}$ the lower values of bond strength obtained for Clearfil SA Cement can be explained by the inability of this cement to remove the smear layer on the dentin surface, as is the case with other self-adhesive resin cements. This leads to the formation of a poor hybridization layer between the resin cement and dentin. Despite the poor interaction of the selfadhesive resin cement with the dentin, it is important to note that this cement showed a good interaction with the zirconia surfaces that had been subjected to either of the two surface treatments. This can be confirmed by the absence of adhesive failures between the self-adhesive resin cement and the ceramic. However, this finding should be further investigated by an experiment designed to evaluate the adhesion between this self-adhesive resin cement and zirconia alone without involving the dentin substrate.

Panavia F showed higher values of shear bond strength, and these could be related to a good dentin hybridization generated by the self-etching adhesive. This adhesive is considered to be a "mild" selfetchant and can remove the smear layer and expose the dentinal tubules. In addition, this adhesive contains the phosphate-based functional monomer 10-MDP, which interacts with collagen and hydroxyapatite components in dentin, resulting in the formation of a strong and stable bond between the resin cement and coronal dentin. ${ }^{27-29}$

Regardless of the resin cements and surface treatments used in the study, the aging process decreased the bond strength. This could be due to the use of the dentin substrate in the study, as it appears to be more susceptible to the hydrolysis. In contrast, even after the thermo-cycling aging process, the interfaces between the resin cements and the zirconia surfaces showed fewer adhesive failures. This is evidence that this interface exhibited stable bonding even after the aging process.

The thickness of the glassy film was found to be approximately $12 \mu \mathrm{m}$ (Figure 2 ). It may be assumed that the film would have no effect on the seating of Y-TZP restorations and their marginal fit. ${ }^{30,31}$ However, future studies should evaluate the real impact of this glassy film on the misfit of Y-TZP frameworks. In addition, the different methods of 
applying the porcelain glaze and zirconia surface treatments before glaze application should also be investigated further. The fracture analysis provided important information about the system behavior. The cement/dentin interface was weaker than the cement/zirconia interface for all experimental groups. Chai and others ${ }^{5}$ cemented zirconia cylinders that had undergone different surface treatments onto a dentin surface and observed a large number of adhesive failures between the cement and dentin. Their results, which are in accordance with the results obtained in this study, highlight the importance of including the dentin substrate in studies on bond strength so that they are more relevant clinically.

\section{CONCLUSION}

Considering the experimental design and the results obtained, we can draw the following conclusions:

1. The bond strength between the resin cements and the zirconia substrate was stronger than that between dentin and the cements, as a function of the efficient conditioning methods performed on the Y-TZP surface, even after lengthy storage.

2. The conventional resin cement containing MDP monomers showed better bond performance than did the self-adhesive resin cement.

3 . The dentin/cement interface appears to be the more critical zone in this system.

4. The application of a thin film of low-fusing glaze porcelain on the zirconia surface followed by hydrofluoric acid etching and silanization appears to offer a promising surface treatment method with which to improve the adhesion between zirconia and resin cement. However, more studies should be performed to confirm this.

\section{Conflict of Interest}

The authors have no proprietary, financial, or other personal interest of any nature or kind in any product, service, and/or company that is presented in this article.

(Accepted 2 October 2012)

\section{REFERENCES}

1. Bottino MA, Valandro LF, Buso L, \& Scotti R (2005) Effect of surface treatments on the resin bond to zirconium-based ceramic International Journal of Prosthodontics 18(1) 60-65.

2. Atsu SS, Kilicarslan MA, Kucukesmen HC, \& Aka PS (2006) Effect of zirconium-oxide ceramic surface treatments on the bond strength to adhesive resin Journal of Prosthetic Dentistry 95(6) 430-436.
3. Ozcan M, Kerkdijk S, \& Valandro LF (2008) Comparison of resin cement adhesion to Y-TZP ceramic following manufactures' instructions of the cements only Clinical Oral Investigation 12(3) 279-282.

4. Cura C, Ozcan M, Isik G, \& Saracoglu A (2011) Comparison of alternative adhesive cementation concepts for zirconia ceramic: Glaze layer VS zirconia primer Journal of Adhesive Dentistry 13(1) 1-8.

5. Chai J, Chu FCS, \& Chow TW (2011) Effect of surface treatment on shear bond strength of zirconia to human dentin Journal of Prosthodontics 20(3) 173-179.

6. Kim MJ, Kim YK, Kim KH, \& Kwon TY (2011) Shear bond strengths of various luting cements to zirconia ceramic: Surface chemical aspects Journal of Dentistry 39(11) 795-803.

7. Valandro LF, Ozcan M, Bottino MC, Scotti R, Bottino MA, \& Della Bona A (2006) Bond strength of a resin cement to high-alumina and zirconia-reinforced ceramics: The effect of surface conditioning Journal of Adhesive Dentistry 8(3) $175-181$.

8. Ozcan M, Alkumru HN, \& Gemalmaz D (2001) The effect of surface treatment on the shear bond strength of luting cement to a glass-infiltrated alumina ceramic International Journal of Prosthodontics 14(4) 335-339.

9. Della Bona A, Donassollo TA, Demarco FF, Barret AA, \& Mecholsky JJ Jr (2007) Characterization and surface treatment effects on topography of a glass-infiltrated alumina/zirconia-reinforced ceramic Dental Materials 23(6) 769-775.

10. Ozcan M, Cura C, \& Valandro LF (2011) Early bond strength of two resin cements to Y-TZP ceramic using MPS or MPS/4-META silanes Odontology 99(1) 62-67.

11. Kern M, \& Wegner SM (1998) Bonding to zirconia ceramic: Adhesion methods and their durability Dental Materials 14(1) 64-71.

12. Ozcan M, \& Valittu PK (2003) Effect of surface conditioning methods on the bond strength of luting cement to ceramics Dental Materials 19(8) 725-731.

13. Friederich R, \& Kern M (2002) Resin bond strength to densely sintered alumina ceramic International Journal of Prosthodontics 15(4) 333-338.

14. Aboushelib MN, Kleverlaan CJ, \& Feilzer AJ (2007) Selective infiltration-etching technique for a strong and durable bond of resin cements to zirconia-based materials Journal of Prosthetic Dentistry 98(5) 379-388.

15. Zhang Y, Lawn BR, Rekow ED, \& Thompson VP (2004) Effect of sandblasting on the long-term performance of dental ceramics Journal of Biomedical Material Researches Part B 71(2) 381-386.

16. Everson P, Addison O, Palin WM, \& Burke FJT (2012) Improved bonding of zirconia substructures to resin using a "glaze-on" technique Journal of Dentistry 40(4) 347-351.

17. Valentino TA, Borges GA, Borges LH, Platt JA, \& Sobrinho-Correr L (2012) Influence of glazed zirconia on dual-cure luting agent bond strength Operative Dentistry 37(2) 181-187.

18. Ntala P, Chen X, Niggli J, \& Cattel M (2010) Development and testing of multi-phase glazes for adhesive 
bonding to zirconia substrates Journal of Dentistry 38(10) $773-781$.

19. Edelhoff D, \& Ozcan M (2007) To what extent does the longevity of fixed dental prostheses depend on the function of cement? Clinical Oral Implants Researches 18(Supplement 3) 193-204.

20. Sailer I, Feher A, Filser F, Luthy H, Gauckler LJ, Scharer P, \& Franz Hämmerle CH (2006) Prospective clinical study of zirconia posterior fixed partial dentures: 3-year follow-up Quintessence International 37(9) 685-693.

21. Peutzfeldt A, Sahafi A, \& Flury S (2011) Bonding of restorative materials to dentin with various luting agents Operative Dentistry 36(3) 266-273.

22. Moon JE, Kim SH, Lee JB, Ha SR, \& Choi YS (2011) The effect of preparation order on the crystal structure of yttria-stabilized tetragonal zirconia polycrystal and the shear bond strength of dental resin cements Dental Materials 27(7) 651-663.

23. Montenegro R, Needleman L, Moles D, \& Tonetti M (2002) Quality of RCTs in periodontology-A systematic review Journal of Dental Research 81(12) 866-870.

24. Bitter K, Paris S, Pfuertner C, Neumann K, \& Kielbassa AM (2009) Morphological and bond strength evaluation of different resin cements to root dentin European Journal of Oral Science 117(3) 326-333.

25. De Munck J, Vargas M, Van Landuyt K, Hikita K, Lambrechts P, \& VanMeerbeek B (2004) Bonding of an auto-adhesive luting material to enamel and dentin Dental Materials 20(10) 963-971.

26. Saskalauskaite E, Tam LE, \& McComb D (2008) Flexural strength, elastic modulus, and $\mathrm{pH}$ profile of self-etch resin luting cements Journal of Prosthodontics 17(4) 262-268.

27. Zicari F, Coutinho E, De Munck J, Poitevin A, Scotti R, Naert I, \& Van Meerbeek B (2008) Bonding effectiveness and sealing ability of fiber-post bonding Dental Materials 24(7) 967-977.

28. Fukegawa D, Hayakawa S, Yoshida Y, Suzuki K, Osaka A, \& Van Meerbeek B (2006) Chemical interaction of phosphoric acid ester with hydroxyapatite Journal of Dental Research 85(10) 941-944.

29. Inoue S, Koshiro K, Yoshida Y, De Munck J, Nagakane K, \& Suzuki K (2005) Hydrolytic stability of self-etch adhesives bonded to dentin Journal of Dental Research 84(12) 1160-1164.

30. Yeo IS, Yang JH, \& Lee JB (2003) In vitro marginal fit of three all-ceramic crown systems Journal of Prosthetic Dentistry 90(5) 459-464.

31. Gonzalo E, Suarez MJ, Serrano B, \& Lozano JF (2009) A comparison of the marginal vertical discrepancies of zirconium and metal ceramic posterior fixed dental prostheses before and after cementation Journal of Prosthetic Dentistry 102(6) 378-384. 